\title{
WEIGHTS IN COHOMOLOGY GROUPS ARISING FROM HYPERPLANE ARRANGEMENTS
}

\author{
MINHYONG KIM
}

(Communicated by Eric M. Friedlander)

\begin{abstract}
The formalism of weights allows very simple analysis of the cohomology of hyperplane complements in a uniform fashion for different cohomology theories. An $l$-adic analogue of Arnold's conjecture on the torsion-freeness of these cohomology groups is one of the consequences.
\end{abstract}

Let $k$ be a field and let $V=\operatorname{Spec}\left(k\left[x_{1}, x_{2}, \ldots, x_{d}\right]\right)$ be an affine $d$-space over $k$. A hyperplane arrangement in $V$ is a collection $\mathscr{A}$ of affine subspaces of codimension 1 in $V$. We are concerned with the hyperplane complement $M(\mathscr{A}):=V-\left(\bigcup_{H \in \mathscr{A}} H\right)$ and its cohomology.

Theorem 1. Let $k=\mathbf{C}$. Then the mixed Hodge structure on $H^{i}(M(\mathscr{A}), \mathbf{Z})$ is pure of weight $2 i$ and Hodge type $(i, i)$.

Theorem $\mathbf{1}^{\prime}$. Suppose the hyperplanes are defined over the finite field $\mathbf{F}_{q}$ and $k$ is an algebraic closure, so that $M(\mathscr{A})=M(\mathscr{A})_{0} \otimes_{\mathbf{F}_{q}} k$. Let $l$ be a prime not dividing $q$. Then (a) the étale cohomology group $H^{i}\left(M(\mathscr{A}), \mathbf{Z}_{l}\right)$ is pure of weight $2 i$, and (b) in fact, the geometric Frobenius $\mathrm{Fr}_{q}$ acts on $H^{i}\left(M(\mathscr{A}), \mathbf{Z}_{l}\right)$ as multiplication by $q^{i}$.

As the reader will see below, the proofs of Theorems 1 and $1^{\prime}$ are rather trivial. In some respects, the first is also just a reformulation of known results. The cohomology of hyperplane complements has been studied extensively by several authors and the structure of the cohomology algebra has been explicitly determined $[8,9]$. Numerous results and conjectures in topology, algebra, and combinatorics arising from this study can be found in the beautiful monograph of Orlik and Terao [9] which also contains a full bibliography. I believe, nonetheless, that Theorem 1 needs to be stated at least once in the formulation above for the following reasons:

(1) The 'philosophy of weights' [1] renders both the statement and the proof of a 'soft' result regarding cohomology simpler and conceptually more transparent than a direct computation;

(2) it reveals an essential underlying structure more basic than so involved an object as the full cohomology algebra; and

Received by the editors June 24, 1992.

1991 Mathematics Subject Classification. Primary 14M99, 14F20.

The author was supported in part by NSF Grant DMS 9106444 .

(c)1994 American Mathematical Society 
(3) it suggests, thereby, analogues for other cohomology theories (e.g., Theorem $1^{\prime}$ ).

To this end, I reproduce below several results of other authors with new proofs from a purely cohomological and elementary standpoint which avoids, in particular, the differential forms and the combinatorial complexes prevalent in [9]. These are then transcribed easily into the setting of Theorem $1^{\prime}$.

In some sense, the work contained in [9] deals with cohomology only in the de Rham realization. It ignores, therefore, the 'Tate twists' which are crucial for the Hodge and $l$-adic theories. But remembering the twists is in fact all that is necessary for proving the theorems above. It leads also to a two-line proof of a fundamental result from [9], namely the splitting of the long exact cohomology sequence associated to the deletion and restriction of an arrangement. I hope this will convince the experts working on hyperplane arrangements that the point of view suggested in this paper is useful. In any case, what follows may at least serve as a 'toy model' for some of the deep and complex ideas surrounding the theory of weights [1-3].

In the second section, we recall a theorem from [9] relating the Betti numbers of a real arrangement to those of its complexification. It was an attempt to understand this relation which led first to Theorem 2 and then to Theorems 1 and 1 ', in that order. The new 'fixed-point formula' proof we present has the advantage of translating immediately to the $l$-adic setting. The effect is a formula showing that $M(\mathscr{A})_{0}\left(\mathbf{F}_{q}\right)$, the number of points of the hyperplane complement in a finite field, is a purely combinatorial invariant; that is, it depends only on the geometric poset $L(\mathscr{A})$ associated to $\mathscr{A}$.

Notation. $\mathbf{Z}(-1)$ is the pure Hodge structure $H^{2}\left(\mathbf{P}^{1}, \mathbf{Z}\right)$ of weight 2 and Hodge type $(1,1) . \mathbf{Z}_{l}(-1)$ is the étale cohomology group $H^{2}\left(\mathbf{P}^{1}, \mathbf{Z}_{l}\right)$ with Frobenius $\mathrm{Fr}_{q}$ acting as multiplication by $q$. If $M$ is a Hodge structure, $M(-1):=$ $M \otimes \mathbf{Z}(-1)$. If $M$ is a $\mathbf{Z}_{l}$ module with Frobenius action, then $M(-1):=$ $M \otimes \mathbf{Z}_{l}(-1)$.

The letter $l$ always denotes a prime distinct from the characteristic of the ground field.

\section{WeIghtS}

The arguments in this section work equally well for the complex case and (algebraic closure of) the finite field case. The cohomology groups will be equipped with mixed Hodge structures or Frobenius actions accordingly and taken with $\mathbf{Z}$ and $\mathbf{Z}_{l}$ coefficients, respectively. Tensor products are then taken over the respective coefficient rings.

If $|\mathscr{A}| \geq 1$, then we can fix an element $H_{0} \in \mathscr{A}$ and define deletion $\mathscr{A}^{\prime}=$ $\mathscr{A}-H_{0}$ and restriction $\mathscr{A}^{\prime \prime}=\left\{H_{0} \cap H \mid H \in \mathscr{A}^{\prime}\right\}$, where the latter is viewed as an arrangement in $H_{0}$. Note that $\left|\mathscr{A}^{\prime}\right|=|\mathscr{A}|-1$, and $\left|\mathscr{A}^{\prime \prime}\right| \leq|\mathscr{A}|-1$.

$M\left(\mathscr{A}^{\prime}\right)$ contains $M(\mathscr{A})$ as an open subspace with complement $M\left(\mathscr{A}^{\prime \prime}\right)$ :

$$
M\left(\mathscr{A}^{\prime \prime}\right) \stackrel{i}{\hookrightarrow} M\left(\mathscr{A}^{\prime}\right) \stackrel{j}{\hookleftarrow} M(\mathscr{A}) .
$$

This gives rise to a long exact cohomology sequence $[4$, p. 95, 7, p. 92]

$$
\begin{aligned}
H_{M\left(\mathscr{A}^{\prime \prime}\right)}^{i}\left(M\left(\mathscr{A}^{\prime}\right)\right) & \rightarrow H^{i}\left(M\left(\mathscr{A}^{\prime}\right)\right) \rightarrow H^{i}(M(\mathscr{A})) \\
& \stackrel{\delta}{\rightarrow} H_{M\left(\mathscr{A}^{\prime \prime}\right)}^{i+1}\left(M\left(\mathscr{A}^{\prime}\right)\right) \rightarrow H^{i+1}\left(M\left(\mathscr{A}^{\prime}\right)\right)
\end{aligned}
$$


preserving Hodge structures and Frobenius actions.

By purity $[2,9.2 .1 .2 ; 7$, VI.5-VI.6], we arrive at the exact sequence

$$
\begin{aligned}
H^{i-2}\left(M\left(\mathscr{A}^{\prime \prime}\right)\right)(-1) & \rightarrow H^{i}\left(M\left(\mathscr{A}^{\prime}\right)\right) \rightarrow H^{i}(M(\mathscr{A})) \\
& \stackrel{\delta}{\rightarrow} H^{i-1}\left(M\left(\mathscr{A}^{\prime \prime}\right)\right)(-1) \rightarrow H^{i+1}\left(M\left(\mathscr{A}^{\prime}\right)\right),
\end{aligned}
$$

again preserving Hodge structures and Frobenius actions.

Proof of Theorem 1. When the cardinality of $\mathscr{A}$ is zero, the statement is trivial. Now, arguing by induction on $|\mathscr{A}|$, we see that the second and fourth terms in (*) have the weight and Hodge type as stated. This gives what we want for the third term, which is trapped between them.

Proof of Theorem $1^{\prime}(a)$. This is word for word the same as the proof of Theorem 1 except for a deletion of the reference to the Hodge type.

Corollary. In both settings, the cohomology groups $H^{i}(M(\mathscr{A}))$ are torsion-free, and the long exact sequence $(*)$ splits into short exact sequences :

$$
0 \rightarrow H^{i}\left(M\left(\mathscr{A}^{\prime}\right)\right) \rightarrow H^{i}(M(\mathscr{A})) \stackrel{\delta}{\rightarrow} H^{i-1}\left(M\left(\mathscr{A}^{\prime \prime}\right)\right)(-1) \rightarrow 0 .
$$

Proof. Using induction on $|\mathscr{A}|$, we may assume that the cohomology groups of $M\left(\mathscr{A}^{\prime}\right)$ and $M\left(\mathscr{A}^{\prime \prime}\right)$ are torsion-free (the case $|\mathscr{A}|=0$ being obvious). By counting weights, we see that the first and the last maps in $(*)$ must be zero when tensored with the rationals. But then the torsion-free assumption allows us to conclude that the maps themselves are zero. This gives us the short exact sequence above which, in turn, let us complete the induction and conclude that $H^{i}(M(\mathscr{A}))$ is torsion-free.

Remarks. (1) This corollary in the complex case is proved in [9] by dislaying an isomorphism with a combinatorially defined complex which splits by construction. It need hardly be said that such an explicit description is of tremendous interest and beauty in its own right as well as useful for many other purposes. The proof above should be regarded merely as a complement.

(2) The torsion-freeness of the étale cohomology groups above can be regarded as an $l$-adic analogue of Arnold's conjecture [9].

The case of the hyperplane $\{0\}$ in affine one-space $\mathbf{A}^{1}$ is elementary but important:

$$
\{0\} \hookrightarrow \mathbf{A}^{1} \hookleftarrow \mathbf{A}^{1}-\{0\}
$$

from which we get

$$
H^{1}\left(\mathbf{A}^{1}-\{0\}\right) \stackrel{\delta}{\simeq} H^{0}(\{0\})(-1) .
$$

Here, $\delta$ is the (natural) boundary map for cohomology.

Given $\mathscr{A}=\left\{H_{i}\right\}_{0}^{k}, \mathscr{A}^{\prime}$ will always denote the deletion with respect to $H_{0}$ and $\alpha_{i}$ will denote a defining linear form for $H_{i}$ so that we have a commutative diagram

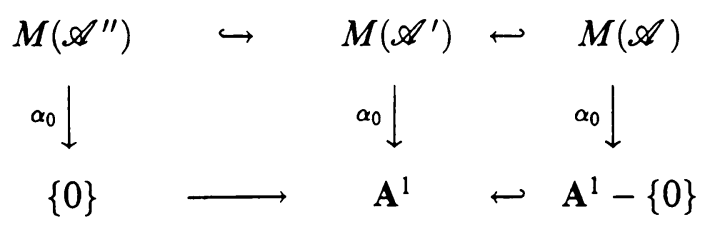


from which we get a commutative square

$$
\begin{array}{ccc}
H^{1}(M(\mathscr{A})) \stackrel{\delta}{\longrightarrow} H^{0}\left(M\left(\mathscr{A}^{\prime \prime}\right)\right)(-1) \\
\alpha_{0}^{*} \uparrow & \alpha_{0}^{*} \uparrow \simeq \\
H^{1}\left(\mathbf{A}^{1}-\{0\}\right) \stackrel{\delta}{\longrightarrow} H^{0}(\{0\})(-1) .
\end{array}
$$

The squares in the following diagram commute as well, and the rows are exact:

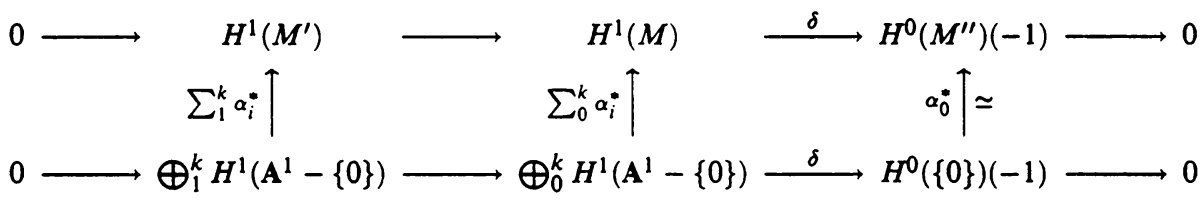

Thus we see by induction on $|\mathscr{A}|$ and the snake lemma that

Proposition 1. The map

$$
\bigoplus_{0}^{k} H^{1}\left(\mathbf{A}^{1}-\{0\}\right) \stackrel{\sum_{0}^{k} \alpha_{i}^{*}}{\longrightarrow} H^{1}(M)
$$

is a surjection.

Similarly, using standard compatibilities between the boundary map and the cup product, we get

0

$\longrightarrow \bigoplus_{1}^{i} H^{1}\left(M^{\prime}\right) \stackrel{1 \times j^{*}}{\longrightarrow}\left(\bigoplus_{1}^{i-1} H^{1}\left(M^{\prime}\right)\right) \oplus H^{1}(M)$

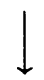

$0 \longrightarrow H^{i}\left(M^{\prime}\right)$

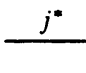

$$
H^{i}(M)
$$

$$
\begin{aligned}
& \stackrel{1 \times \delta}{\longrightarrow}\left(\bigoplus_{1}^{i-1} H^{1}\left(M^{\prime}\right)\right) \oplus H^{0}\left(M^{\prime \prime}\right)(-1) \longrightarrow 0 \\
& \stackrel{(-1)^{i-1} \delta}{\longrightarrow} \quad H^{i-1}\left(M^{\prime \prime}\right)(-1)
\end{aligned}
$$

where the squares are again commutative and the rows are exact. The vertical arrows are induced by the cup product, and hence, are multilinear. On the other hand, the upper row of the diagram preceding Proposition 1 gives us the exact sequence

$$
0 \rightarrow \bigwedge^{i} H^{1}\left(M^{\prime}\right) \rightarrow \bigwedge^{i} H^{1}(M) \rightarrow \bigwedge^{i-1} H^{1}\left(M^{\prime}\right) \otimes H^{0}\left(M^{\prime \prime}\right)(-1) \rightarrow 0
$$

since $H^{0}\left(M^{\prime \prime}\right)(-1) \simeq \mathbf{Z}(-1) \quad\left(\right.$ or $\left.Z_{l}(-1)\right)$ canonically. Composing the last map with $i^{*} \otimes 1$ and using the above-mentioned compatibility finally gives us:

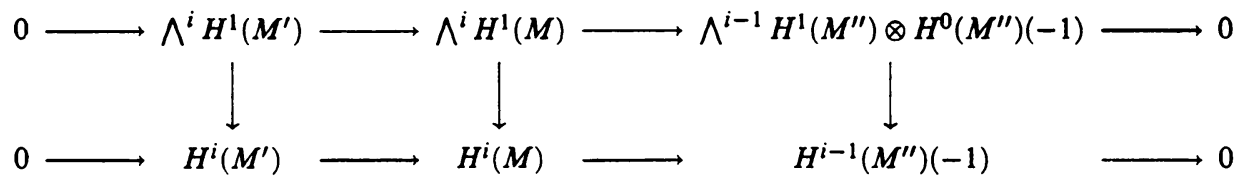

Here, the upper row is not exact in the middle, but a standard diagram chase and yet another induction on $|\mathscr{A}|$ still gives us (cf. [9, Chapter 5] for the complex case) 
Proposition 2. The cup product induces an epimorphism

$$
\bigwedge^{i} H^{1}(M(\mathscr{A})) \rightarrow H^{i}(M(\mathscr{A})) .
$$

Propositions 1 and 2 in conjunction imply Theorem $1^{\prime}(b)$.

\section{BETTI NUMBERS}

Let $k$ be the real numbers and suppose therefore that the $\alpha_{i}$ are defined over $\mathbf{R}$. We can then consider the complexification $M(\mathscr{A})$ c with the continuous action of complex conjugation. Then

Theorem 2. The Galois group of $\mathbf{C}$ over $\mathbf{R}$ acts on the cohomology $H^{i}\left(M(\mathscr{A})_{\mathbf{C}}\right)$ as multiplication by $(-1)^{i}$.

Proof. The long exact cohomology sequences are equivariant with respect to the action of complex conjugation, as are the maps $\alpha_{i}^{*}$ (since they are defined over R). So the theorem follows by induction or by Propositions 1 and 2 .

Remarks. (1) $\mathrm{Gal}(\mathbf{C} / \mathbf{R})$ acts compatibly on both the singular cohomology and the étale cohomology. The proof clearly works for either.

(2) Phrasing such a simple theorem as a result about Galois representations may appear somewhat pedantic. It is in keeping, however, with the spirit of the introduction, and suggests that one should look for analogues in the theory of $p$-adic periods [5]. In this connection, the prevalence of de Rham cohomology in the theory developed by [9] should be kept in mind.

Recall the following formula from $\left[9\right.$, p. 196] for the Betti numbers $b_{i}$ of $M(\mathscr{A})$ and its complexification:

$$
\sum b_{i}(M(\mathscr{A})(\mathbf{R}))=\sum b_{i}(M(\mathscr{A})(\mathbf{C})) .
$$

Since the real variety is just a disjoint union of contractible spaces, the sum on the left actually collapses to the single term $b_{0}(M(\mathscr{A}))$.

The formula may be proved by utilizing a 'fixed-point' formula for the action of a finite cyclic group $G$ with generator $\gamma$ acting smoothly on a manifold $X$ $[10$, p. 225]:

$$
\chi\left(X^{G}\right)=\sum_{i}(-1)^{i} \operatorname{Tr}\left(\gamma^{*}: H^{i}(X) \rightarrow H^{i}(X)\right) .
$$

The cohomology here and henceforward will be taken with $\mathbf{Q}$ or $\mathbf{Q}_{l}$ coeffcient. $\chi$ denotes the Euler characteristic and $X^{G}$ the fixed-point set of the action. The formula is usually stated for compact manifolds, but easily generalizes to our setting of complex conjugation acting on $M(\mathscr{A})(\mathbf{C})$, where we have merely removed compact submanifolds stable under the group action (with stable tubular neighorhoods, since the group is finite) from a compact manfiold. Theorem 2 and this argument immediately yield the formula for the Betti numbers, since $\operatorname{Tr}\left(\gamma^{*} \mid H^{i}(M(\mathscr{A})(\mathbf{C}))\right)=(-1)^{i} b_{i}(M(\mathscr{A})(\mathbf{C}))$ while the fixed point set is just $M(\mathscr{A})(\mathbf{R})$.

To see the finite field analogue, that is, to continue the setting of Theorem $1^{\prime}$, where $M(\mathscr{A})=M(\mathscr{A})_{0} \otimes k$, we recall the Grothendieck-Lefschetz trace formula [7, Theorem VI.13.4]:

$$
\left|M_{0}\left(\mathbf{F}_{q}\right)\right|=\sum_{i}(-1)^{i} \operatorname{Tr}\left(\operatorname{Fr}_{q}: H_{c}^{i}\left(M_{0} \otimes_{\mathbf{F}_{q}} k\right) \rightarrow H_{c}^{i}\left(M_{0} \otimes_{\mathbf{F}_{q}} k\right)\right) .
$$


By Poincaré duality $\left[7\right.$, p. 276], $H_{c}^{i} \simeq\left(H^{2 d-i}\right)^{*}(-d)$, and Theorem $1^{\prime}(\mathrm{b})$, we arrive at

Theorem 3. $\left|M(\mathscr{A})_{0}\left(\mathbf{F}_{q}\right)\right|=\sum_{i}(-1)^{i} q^{i} b_{2 d-i}(M(\mathscr{A}))$.

Recall from [9] the poset $L(\mathscr{A})$ associated to $\mathscr{A}$ : The objects are given by the nonempty intersections of elements of $\mathscr{A}$ together with the element $V$. The partial ordering is defined by

$$
X \leq Y \text { iff } Y \subset X
$$

so that $V$ is the unique minimal element of $L(\mathscr{A})$. There is a rank function $r(X):=\operatorname{codim}(X)$ making $L(\mathscr{A})$ into a geometric poset $[9$, p. 24]. The Poincare polynomial of $\mathscr{A}$ is defined by the formula

$$
\pi(\mathscr{A}, t):=\sum_{X \in L(\mathscr{A})} \mu(X)(-t)^{r(X)},
$$

where $\mu$ is the Möbius function of $L(\mathscr{A})$ characterized by

$$
\mu(V)=1, \quad \sum_{Y \leq X} \mu(Y)=0, \quad \text { all } X \in L(\mathscr{A}) .
$$

It is known ([8] in the complex case, [6] in general) that

$$
\pi(\mathscr{A}, t)=\sum_{i} \operatorname{dim} H^{i}\left(M(\mathscr{A}), \mathbf{Q}_{l}\right) t^{i}
$$

that is, the topological Poincare polynomial agrees with the combinatorial one. In any case, it can easily be derived again using the inductive relation $[9$, p. 46]

$$
\pi(\mathscr{A}, t)=\pi\left(\mathscr{A}^{\prime}, t\right)+t \pi\left(\mathscr{A}^{\prime \prime}, t\right)
$$

and the corresponding relation for the topological Poincare polynomials implied by Corollary 1. (This is the argument in [9].) This says that the etale Betti numbers of $M(\mathscr{A})$ are purely combinatorial invariants. That is they depend only on the partially ordered set $L(\mathscr{A})$ associated to $\mathscr{A}$ equipped with its rank function. An upshot of Theorem 3 is that the same is true of the diophantine invariant $\left|M(\mathscr{A})_{0}\left(\mathbf{F}_{q}\right)\right|$. Kai Beherend has pointed out to me that this is not too difficult to see by a direct argument.

Note. It has been called to my attention that similar results have been obtained by Lehrer in the Bulletin of the London Mathematical Society, vol. 24 (1992). But his results seem to deal only with rational coefficients. In particular, the corollary to Theorem $1^{\prime}$ (the $l$-adic Arnold's conjecture) is not covered by his results. Also, the referee has called to my attention the fact that Theorem 1 in the rational case has been obtained independently (and earlier) in a manuscript of Shapiro (to be published). It is hoped, however, that the parallel formulation of the mixed Hodge-structure and the étale cohomology case presented in this paper will be of some interest. It is my understanding that Shapiro's paper also does not deal with integral information (i.e., application to Arnold's conjecture).

\section{ACKNOWLEDGMENT}

I am grateful to Peter Orlik for having aroused my interest in this subject and to Bill Dwyer for a very useful conversation regarding the material in $\S 2$. 


\section{REFERENCES}

1. P. Deligne, Théories de Hodge. I, Actes du Congrès International des Mathématiciens, Nice, vol. 1, 1970, pp. 425-430.

2. __ Théories de Hodge. II, III, Inst. Hautes Études Sci. Publ. Math 40 (1972), 5-57; 44 (1975), 6-77.

3. __ Poids dans la cohomologie des variétés algébriques, Proc. Internat. Congr. Math. (Vancouver), Canad. Math. Congress, vol. 1, 1974, pp. 79-85.

4. F. Elzein, Mixed Hodge structures, Trans. Amer. Math. Soc. 275 (1983), 71-106.

5. L. Illusie, Cohomologie de de Rham et cohomologie étale p-adic, Astérisque 189-190 (1990 Canadian Math. Congress), 325-374.

6. M. Kim, On Poincaré polynomials for hyperplane arrangements in positive characteristic, Comm. Algebra 21 (1993), 1337-1346.

7. J. Milne, Étale cohomology, Princeton Univ. Press, Princeton, NJ, 1980.

8. P. Orlik and L. Solomon, Combinatorics and topology of complements of hyperplanes, Invent. Math. 56 (1980), 167-189.

9. P. Orlik and H. Terao, Arrangements of hyperplanes, Grundlehren Math. Wiss., vol. 300, Springer-Verlag, Berlin, Heidelberg, and New York, 1992.

10. T. tom Dieck, Transformation groups, de Gruyter Verlag, Berlin and New York, 1987.

Department of Mathematics, Massachusetts institute of Technology, Cambridge, MASSACHUSETTS 02139

E-mail address: mhkim@math.mit.edu

Current address: Department of Mathematics, Columbia University, New York, New York 10027

E-mail address: kim@math.columbia.edu 